\title{
Design of an Intelligent Voice Controlled Home Automation System
}

\author{
Sonali Sen, Shamik Chakrabarty, Raghav Toshniwal, Ankita Bhaumik \\ Department of Computer Science \\ St. Xavier's College, Kolkata
}

\begin{abstract}
Automation is a trending topic in the 21 st century making it play an important role in our daily lives. The main attraction of any automated system is reducing human labour, effort, time and errors due to human negligence. With the development of modern technology, smart phones have become a necessity for every person on this planet. Applications are being developed on Android systems that are useful to us in various ways. Another upcoming technology is natural language processing which enables us to command and control things with our voice. Combining all of these, our paper presents a micro controller based voice controlled home automation system using smartphones. Such a system will enable users to have control over every appliance in his/her home with their voice. All that the user needs is an Android smartphone, which is present in almost everybody's hand nowadays, and a control circuit. The control circuit consists of an Arduino Uno microcontroller, which processes the user commands and controls the switching of devices. The connection between the microcontroller and the smartphone is established via Bluetooth, a widespread wireless technology used for sharing data.
\end{abstract}

\section{General Terms}

Voice recognition

\section{Keywords}

Arduino Uno, HC-05 Bluetooth Module, Home Automation, Smartphone, Voice Control

\section{INTRODUCTION}

The foremost aim of technology has been to increase efficiency and decrease effort. With the advent of 'Internet of Things' in the last decade, we have been pushing for ubiquitous computing in all spheres of life. It thus is of extreme importance to simplify human interfacing with technology. Automation is one such area that aims that achieves simplicity whilst increasing efficiency. Voice controlled House Automation System aims to further the cause of automation so as to achieve the goal of simplicity [1] [4].

The primitive man realized that an effective way to communicate with one another is through voice. With minimum effort, ideas could be narrated with relative ease. When the first computers came around, achieving the level of sophistication so as to narrate commands using voice to a machine was only realized in science fiction. However with tremendous breakthroughs in the field, we are at the precipice of truly using voice to interface with devices.
Using this effective yet ingrained form of communication we would humanize technology to a great extent. Voice controlled House Automation System deploys the use of voice to control devices [8].

The advantages of using voice as an interfacing medium are multifold. Firstly we would do away with or significantly decrease the need of training for operating technology. Secondly, the simplification of services would entail a wider adoption of existing technology and would help people with varied disabilities access the same technology. We have deployed an Android Application as user front end primarily because of the ease at which the platform provides us with means to use complex technology and due to the widespread adoption in the mobile industry. Android is being used as the operating system for over $80 \%$ of the smartphones.

Voice controlled House Automation System leverages the power of Arduino to provide a holistic voice controlled automation system. Using Natural Language Processing and the available hardware in most smartphones, it translates voice to be used for controlling electrical devices.

\section{SYSTEM DESIGN}

\subsection{System Components}

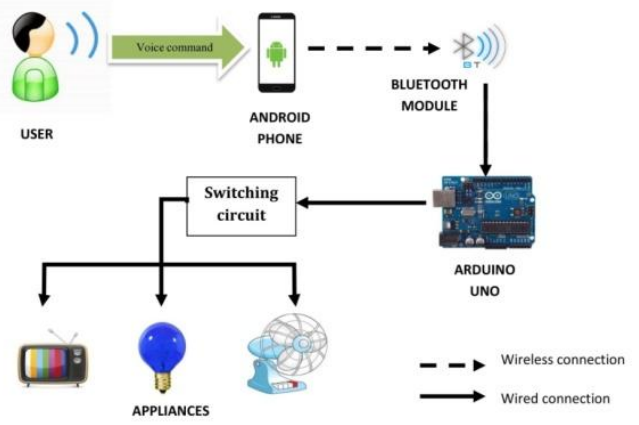

Figure 1: Block Diagram of the System

The Voice-operated Android and Arduino Home automation system uses an Android based Bluetooth enabled phone for its application and the Arduino Uno as the microcontroller. The key components of this system are: 
- Android based phone

- Bluetooth module

- Arduino Uno

- Relay boards

\subsubsection{Android Based Phone}

Android is a mobile operating system (OS) based on the Linux kernel and currently developed by Google. With a user interface based on direct manipulation, the OS uses touch inputs that loosely correspond to real-world actions, like swiping, tapping, pinching, and reverse pinching to manipulate on-screen objects, and a virtual keyboard. We have used the Android platform because of its huge market globally and it's easy to use user interface [5]. Applications on the Android phones extend the functionality of devices and are written primarily in the Java programming language using the Android software development kit (SDK). The voice recognizer which is an in built feature of Android phones is used to build an application which the user can operate to automate the appliances in his house. The user interface of the application is shown below:

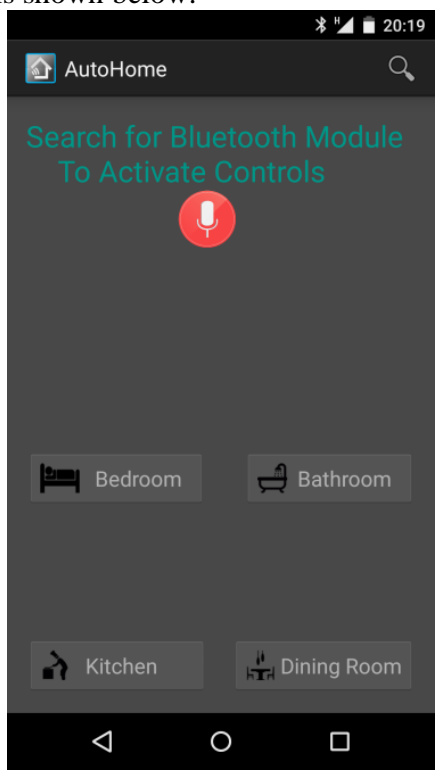

Figure 2: Interface for the Voice Control Application

The microphone button is tapped and the voice command is given to switch the corresponding device on/off. The voice recognizer listens and converts what is said to the nearest matching words or text. The Bluetooth adapter present in the phone is configured to send this text to the Bluetooth module on the Arduino Uno board that would in turn control the electrical appliances through the relay boards.

\subsubsection{Bluetooth Module}

Bluetooth is a wireless technology standard for exchanging data over short distances (using short-wavelength UHF radio waves in the ISM band from 2.4 to $2.485 \mathrm{GHz}$ ) from fixed and mobile devices, and building personal area networks (PANs) [7]. The Bluetooth module being used allows us to transmit and receive signals [5]. It receives the text from the Android phone and transmits it to the serial port of the Arduino Uno.

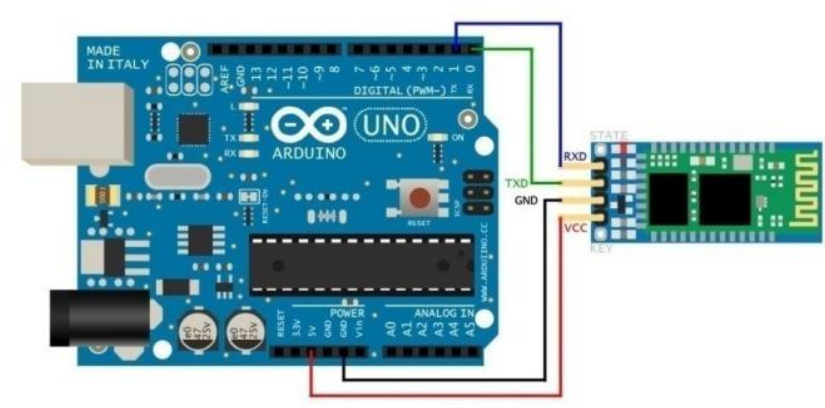

Figure 3: HC-05 connectivity with Atmega328p

The Bluetooth module being used here is the HC-05 module. It is an easy to use Bluetooth SPP (Serial Port Protocol) module, designed for transparent wireless serial connection setup. Serial port Bluetooth module is fully qualified Bluetooth V2.0+EDR (Enhanced Data Rate) $3 \mathrm{Mbps}$ Modulation with complete $2.4 \mathrm{GHz}$ radio transceiver and baseband. It uses CSR Bluecore 04-External single chip Bluetooth system with CMOS technology and with AFH (Adaptive Frequency Hopping Feature). It has a slave default Baud rate of 9600. It auto connects to the last device on power as default. Pairing pin code is " 1234 " as default [3].

\subsubsection{Arduino Uno}

The Arduino Uno is a microcontroller board based on the ATmega328p. It has 14 digital input/output pins (of which 6 can be used as PWM outputs), 6 analog inputs, a 16 $\mathrm{MHz}$ ceramic resonator, a USB connection, a power jack, an ICSP header, and a reset button [2]. It contains everything needed to support the microcontroller. We either need to connect it to a computer using a USB cable or power it with an AC-to-DC adapter. The Arduino circuit acts as an interface between the software part and the hardware part of the project [1].

The Bluetooth module transmits the text to the Arduino Uno serial port. The text is matched against the various combinations of predefined texts to switch the appliances on/off. The appliance name and a command for on/off are stored as predefined command. For example, to switch on a television the user needs to say "television on" and to switch it off he needs to say "television off". The appliances are connected via the relay boards to pin numbers 2,3 and 4 of the Arduino Uno. When the matching text is detected the corresponding pin number is given a high or low output signal to switch the appliance on and off respectively.

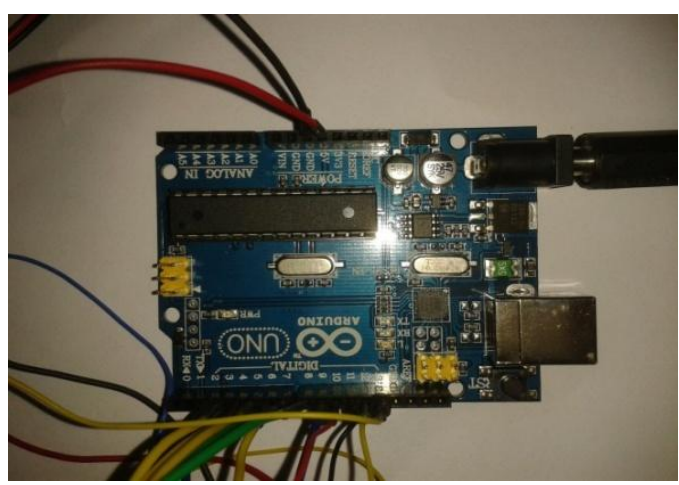

Figure 4: Arduino Uno 


\subsubsection{Relay Boards}

A relay is an electromagnetic switch. In other words it is activated when a current is applied to it. Normally a relay is used in a circuit as a type of switch (as shown below). There are different types of relays and they operate at different voltages. When a circuit is built the voltage that will trigger it has to be considered. In this project the relay circuit is used to turn the appliances on/off. The high/low signal is supplied from the Arduino Uno microcontroller. When a low voltage is given to the relay of an appliance it is turned off and when a high voltage is given it is turned on. The relay circuit to drive four appliances in the Voiceoperated Android and Arduino Home automation system is shown below. The number of appliances can be modified according to the user's requirements.

\section{IMPLEMENTATION}

Using the above mentioned components we implement our system on a breadboard. The microcontroller device with the Bluetooth module and relay circuit needs to be attached with the switch board. Then we need to launch the android based application-"AutoHome" on our Smartphone. Through the application we can instruct the microcontroller to switch on/off an appliance. After getting the instruction through the Bluetooth module the microcontroller gives the signal to the relay board.

The application first searches for the Bluetooth device. If it is available then it launches the voice recognizer. It reads the voice and converts the audio signal into a string. It produces a value for each appliance which will be given to the microcontroller device. The microcontroller uses the port in serial mode. After reading the data it decodes the input value and sends a signal to the parallel port through which the relay circuit will be activated.

In this work we use Bluetooth module. We can also attach a GSM module to do the work, using which the application can be used anywhere where a mobile network is available.

Some images to illustrate the working of the system have been given below.

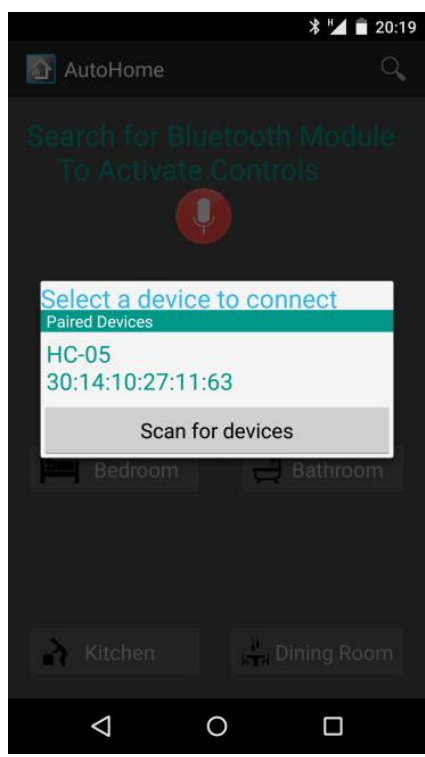

Figure 5: Application connecting to the Bluetooth device

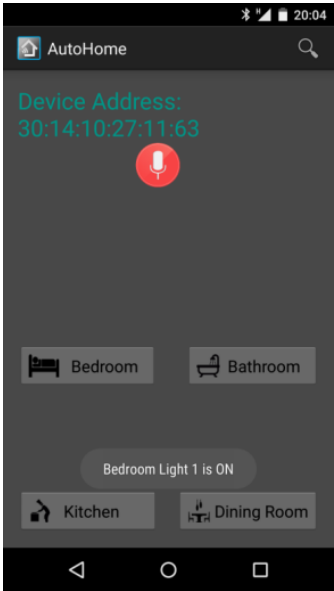

Figure 6: Turning ON Bedroom Light 1

\section{FLOWCHART}

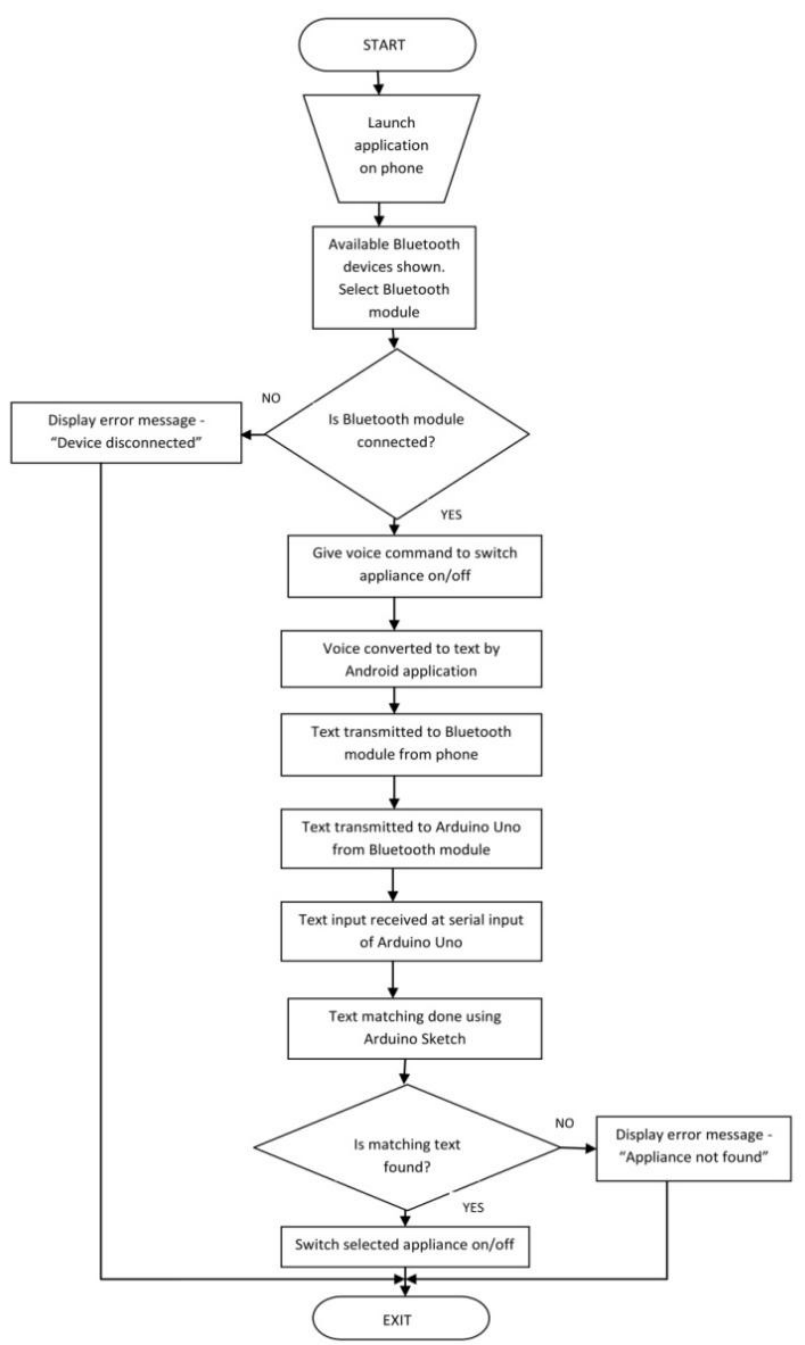

Figure 7: Flowchart of the entire system

\section{CONCLUSION}

The proposed project undertakes a viable solution the need of automation at the very basic level, that is, in our homes. The project will enable us to bring every appliance at every corner of our home under our control from a single 
point without having to get up and manually switch on or off the appliance. The use of a Bluetooth module assists the use of this system from various locations in our house.

The system is further simplified by allowing appliances to be controlled by our voice. The user need not have to have to immense knowledge over the language of English. Just by saying the appliance name and the corresponding number assigned to that particular appliance, and telling it to switch on or off will enable the user to have complete control over any appliance without any effort.

Android applications are very simple and user friendly allowing the user to understand its functionalities in very little time. Hence, the use of android application in this system allows a user to easily learn the process and get accustomed to the functions. Moreover, the entire system is very flexible and scalable. Any number of appliances can be added as and when required. Hence, the systems finds use not only in houses but also in many offices where appliances such as fans or lights on multiple floors can be controlled by a person on any of the floors, saving manual labour and human effort to switch on or off the electronic appliances, thereby saving time.

This system, though primarily aimed to reduce human effort, will be of much importance to old aged people and physically handicapped people. It will enable them to control their home devices with ease, without going through much pressure or stress of moving about.

Due to the inexpensive materials used in the construction and further cost optimization if the device is taken to the market, it finds application in a wide area. Scalability of the project would be considerably easier as the device can be used in every building using electrical appliances and devices.

In addition, there have been many advertisements broadcasted by the Government of India promoting awareness to switch off household appliances when not in use and thus save electricity. Hence, such a project would assist the initiatives taken by the government, as most people forget to switch off home appliances and are too lazy to return and switch it off.

\section{ACKNOWLEDGMENTS}

We would like to thank the Principal \& Computer Science Department of St. Xavier's College, Kolkata (Autonomous) for supporting us in this work.

\section{REFERENCES}

[1] Mohamed Abd El-LatifMowad, Ahmed Fathy, Ahmed Hafez "Smart Home Automated Control System Using Android Application and Microcontroller" International Journal of Scientific \& Engineering Research, Volume 5, Issue 5, May-2014 ISSN 2229 5518

[2] Arduino Uno Projects: http://arduino.cc/en/Main/arduinoBoardUno

[3] Datasheet Bluetooth to Serial Port Module HC05: http://www.electronica60norte.com/mwfls/pdf/newB1 uetooth.pdf

[4] Armando Roy Delgado, Rich Picking and Vic Grout "Remote- Controlled Home Automation Systems with Different Network Technologies" Centre for Applied Internet Research (CAIR), University of Wales, NEWI, Wrexham, UK

[5] Ming Yan and Hao Shi "SMART LIVING USING BLUETOOTH BASED ANDROID SMARTPHONE" International Journal of Wireless \& Mobile Networks (IJWMN) Vol. 5, No. 1, February 2013 DOI : 10.5121/ijwmn.2013.5105 65

[6] Folea, S. ; Autom. Dept., Tech. Univ. of Cluj-Napoca, Cluj-Napoca, Romania ; Bordencea, D. ; Hotea, C. ; Valean, H - "Smart home automation system using Wi-Fi low power devices" Published in: Automation Quality and Testing Robotics (AQTR), 2012 IEEE International Conference on Date of Conference: 2427 May 2012 Page(s): 569 - 574 Print ISBN:978-14673-0701-7 ; INSPEC Accession Number: 12853582 Conference Location :Cluj-Napoca ; DOI:10.1109/AQTR.2012.6237775 ; Publisher: IEEE

[7] Chakradhar, B., KrishnaveniI, S., and Naresh , D. 2013. "Bluetooth Based Home Automation and Security System Using ARM9", International Journal of Engineering Trends and Technology (IJETT), Vol. 4 Issue 9, Pg 4053-4058

[8] Obaid, T. et al. 2014. "ZIGBEE BASED VOICE CONTROLLED WIRELESS SMART HOME SYSTEM", International Journal of Wireless \& Mobile Networks (IJWMN), Vol. 6, No. 1, Pg. 47-5 